\title{
« Ne nous engageons point dans les querelles » : un projet de guerre perpétuelle ?
}

\section{Kate E. Tunstall*}

* Kate E. Tunstall, née en 1970, est professeure associée dans la Faculty of Modern Languages à l'Université d'Oxford, où elle enseigne la langue et la littérature françaises. Spécialiste des Lumières, elle a publié Blindness and Enlightenment. An Essay (2010), et a co-traduct (avec Caroline Warman) Le neveu de Rameau (2014). Adresse: Worcester College, University of Oxford, Oxford OX12HB (kate.tunstall@mod-langs.ox.ac.uk). http://www.mod-langs.ox.ac.uk/people/kate-tunstall

Résumé : Cet article aborde la querelle comme un élément de la stratégie d'un philosophe visà-vis de la postérité. On pourrait évidemment penser à des querelleurs invétérés comme Pascal, Voltaire ou Rousseau, mais il sera question ici du cas plus complexe de Diderot et du dernier ouvrage publié de son vivant, l'Essai sur les règnes de Claude et de Néron et sur les mœurs et les écrits de Sénèque pour servir à l'introduction de la lecture de ce philosophe (1782). L'article démontre que celui-ci constitue un cas de «texte-querelle », c'est-à-dire un texte qui met en scène la querelle menée à son propre sujet et qui ainsi la fait redémarrer ; il analyse la posture qu'adopte le philosophe-querelleur, surtout dans l'énoncé paradoxal «ne nous engageons point dans les querelles », dont il examine les qualités performatives.

MOTS-CLES :

Querelle, Éthique, Postérité, Philosophie, Diderot

Summary : This article considers the extent to which a quarrel might be a strategic activity for a writer or philosopher making a bid for posterity. Inveterate quarrellers like Voltaire or Rousseau come to mind, but the case to be examined here is the far less straightforward case of Diderot and in particular the stance of 'accidental quarreller' that he fabricates in his Essai sur les règnes de Claude et de Néron et sur les mours et les écrits de Sénèque pour servir à l'introduction de la lecture de ce philosophe (1782), the last work he published in his lifetime. The article argues that the Essai constitutes what might be called a «quarrel-text », that is to say a text that stages the quarrel of which it is itself the object and, in so doing, reignites it ; it analyses the stance adopted by the philosopher-quarreller as it is revealed in the paradoxical utterance «ne nous engageons point dans les querelles », the performative qualities of which are explored in detail.

\section{AGON à l'agonie}

L'injonction qui sert de titre à mon propos, " ne nous engageons point dans les querelles », est tirée d'un ouvrage paru en 1782, intitulé Essai sur les règnes de Claude et de Néron et sur les mœurs et les écrits de Sénèque pour servir à l'introduction de la lecture de ce philosophe ${ }^{1}$. Il s'agit d'un des derniers livres de Denis Diderot, le dernier publié de son vivant; ce sera mon principal objet d'étude ici. Pourquoi ?

Longtemps négligé par la critique pour des raisons sur lesquelles je reviendrai (mais dont l'une consiste en ce simple fait qu'il s'agit de 600 pages d'histoire romaine), cet ouvrage connaît aujourd'hui un certain regain d'intérêt, du moins chez les dix-huitièmistes ${ }^{2}$. Le

\footnotetext{
${ }^{1}$ Diderot, 1782 , t. 2 , p. 15.

2 Voir Vincent, 2014, p. 491-622 ; MASSEAU, 2012 ; NouIS, 2010 ; CANDLER HAYeS, 2009 ; StALNAKER, 2009 ; LECA-TSIOMIS, 2004 ; CABANE, 2004 ; MALL, 2004 ; MALL, 2000. Il est étrange que le regain d'intérêt pour ce texte ne soit pas repertorié dans FOWLER, 2013.
} 
programme de l'agrégation de lettres modernes y est sans doute pour quelque chose ; non pas que l'Essai y ait figuré, mais les agrégatifs de 2004 avaient un texte de Jean-Jacques Rousseau, à savoir Rousseau, juge de Jean-Jacques (1772-5, publ. posthume 1782), avec lequel l'Essai n'est pas sans rapport ${ }^{3}$. Mais on peut dire aussi, et de manière plus générale, que la critique littéraire s'intéresse depuis quelques années aux dernières œuvres des grands écrivains, à ce que Theodor Adorno a appelé «le style tardif», au genre « testamentaire », aux «afterlives »et à la notion de postérité ${ }^{4}$. Cet intérêt n'est sans doute pas sans rapport avec des phénomènes socio-politiques actuels comme le déclin de l'influence mondiale de la culture européenne et occidentale, mais sur le plan plus restreint du monde intellectuel français, ce n'est pas par hasard, me semble-t-il, que cet intérêt pour le tardif a suivi la disparition dans les années 1980 et 1990 de toute une génération de philosophes français, dont Roland Barthes, Louis Althusser, Michel Foucault, Gilles Deleuze, de ceux que Jacques Derrida, qu'ils ont devancé, a appelés ses « amis ${ }^{5}$ ». Or, dans les années 1770 et 1780, c'est une autre grande génération de philosophes français (ou francophones) qui disparait ; c'est même la génération des Philosophes qui disparaît - Voltaire et Rousseau meurent en 1778, Diderot en 1784. L'Essai sur les règnes de Claude et de Néron et sur les mours et les écrits de Sénèque de Diderot, qui a survécu aux deux autres, constitue son testament intellectuel, moral et littéraire ${ }^{6}$, dans lequel il médite, comme le fera aussi Derrida, sur le thème de l'amitié et, surtout, sur la postérité, la sienne et celle de la philosophie des Lumières. L'Essai est riche en échos pour tous ceux qui s'intéressent à la fortune de cette figure marquante dans la culture française, celle du philosophe, de l'intellectuel, et surtout à la manière dont des philosophes ont eux-mêmes imaginé ou tenté de construire leur propre postérité.

Mais pourquoi privilégier l'Essai comme objet d'étude dans le cadre du projet «AGON ${ }^{7}$ ? Ce n'est pas seulement pare que le projet touche à sa fin, qu'il agonise, et que nous sommes confrontés à la question de savoir quel sera le legs intellectuel de ces cinq ans de travail collectif sur les disputes et les querelles ; c'est aussi, et principalement, parce que l'Essai traite, précisément, des querelles et de leurs effets possibles sur la postérité d'un philosophe, et que l'Essai est lui-même un « texte-querelle ». Je m'explique.

\section{Un « texte-querelle »}

L'Essai qui parut en 1782 est la réponse à une querelle que l'ouvrage avait lui-même déclenchée lors de sa première édition, parue trois ans auparavant, à la toute fin de 1778, sous un titre légèrement différent, Essai sur la vie de Sénèque le philosophe, sur ses écrits et sur les règnes de Claude et de Néron ${ }^{8}$. Cette version, que je nommerai l'Essai-78, parut à Paris chez les frères De Bure avec approbation et privilège, et constituait, même s'il se vendait séparément, le tome sept des Euvres de Sénèque, traduites par Lagrange et parues chez le même éditeur un mois plus tôt la même année 9 .

\section{La querelle de l'Essai-78}

\footnotetext{
${ }^{3}$ NOUIS, 2010 ; GATEFIN, 2007 ; CITTON, 2004 ; FOUCAULT, 1962.

${ }^{4}$ Goulemot, 2012 ; Hinde Stewart, 2010 ; Goodman et Harris, 2018 ; Lilti, 2014 ; Bonnet, 1998 ; BONNET, 1992.

${ }^{5}$ Derrida leur a dédié des pages avant de mourir à son tour en 2004 ; voir DERRIDA, 2003 et 2004.

${ }^{6}$ Voir STALNAKER, 2009 ; EHRARD, 1995, p. 6. L'essayiste affirme que « celui qui conseilla au philosophe de laisser un testament de mort, eut une idée utile et grande », DIDEROT, 1782, t. 2, p. 25.

${ }^{7}$ Pour d'informations sur le projet, voir http://www.agon.paris-sorbonne.fr

${ }^{8}$ Diderot, 1778.

${ }^{9}$ SÉNÈQUe, 1778.
} 
Les premiers comptes rendus de l'Essai-78, parus dans la Correspondance littéraire ${ }^{10}$ et le Mercure de France en décembre $1778^{11}$, étaient plutôt positifs, même élogieux dans le cas du Mercure, dont l'article est de Marmontel; mais le mois de janvier 1779 foisonne de mauvaises critiques, dont deux articles dans le Journal de littérature, des sciences et des arts $^{12}$, deux lettres dans l'Année littéraire du 9 et du 19 janvier ${ }^{13}$, un compte rendu dans les Affiches, annonces et avis divers du 20 janvier $^{14}$, ainsi que celui du Journal de Paris du 25 janvier ${ }^{15}$. Arrive le mois de février et on voit bien que l'on a affaire à une querelle : le Journal encyclopédique de Pierre Rousseau donne un compte rendu positif en deux livraisons, le $1^{\mathrm{er}}$ et le 15 février ${ }^{16}$; le Journal de Paris du 2 février imprime une lettre anonyme qui s'oppose aux critiques du compte rendu du 25 janvier $^{17}$, suivie dans le même numéro d'une réponse des éditeurs $^{18}$; le Mercure de France du 15 février publie une lettre de Dominique Joseph Garat, jeune admirateur de Diderot, qui s'adresse aux éditeurs du Journal de Paris en réponse à leur compte rendu du 25 janvier $^{19}$; le Journal de Paris ne lui répond pas directement mais publie le 6 avril, sous la forme d'une lettre signée «BRA », un deuxième compte rendu de l'Essai à nouveau critique ${ }^{20}$. Puis silence.

\section{L'Essai-82 comme texte-querelle}

Le silence dura trois ans. En février 1782 paraît une deuxième édition de l'Essai. Portant un titre légèrement remanié et sans aucun nom d'éditeur, avec un lieu de publication qui est faux, «Londres » (il s'agit, en réalité, de Bouillon de la société typographique de Pierre Rousseau) : ce que j'appellerai l'Essai-82 propose aux lecteurs une sorte de mise en scène de la querelle à son propre sujet. Il cite les critiques et les éloges qui avaient été adressés à la première édition, et leur répond. Et la querelle redémarre : si le Journal encyclopédique du 15 mars est de nouveau favorable ${ }^{21}$, en novembre, l'Année littéraire est encore acerbe ${ }^{22}$. En mai, Diderot écrit au lieutenant de police, Le Noir, pour lui exprimer son repentir d'avoir publié son $E_{s s a i}{ }^{23}$. Le texte-querelle est donc un objet double : il met en scène une querelle menée à son propre sujet, et sa mise-en-scène constitue une intervention qui réactive la querelle et qui, une fois redémarrée, exigera une nouvelle mise-en-scène. Il s'agit donc d'un supplément à la querelle et d'un supplément de querelle.

C'est la nature de "texte-querelle » de l'Essai-82 qui explique, en bonne partie, me semble-t-il, sa relative obscurité aujourd'hui. Sa forme est complexe, voire compliquée : la citation des critiques introduit une déroutante multiplicité de voix dans un texte qui était déjà loin d'être univoque. La Correspondance littéraire observe en mars 1782 :

\footnotetext{
${ }^{10}$ GRIMM, 1880.

${ }^{11}$ MARMONTEL, 1778.

${ }^{12}$ ANONYME, janvier 1779 ; ANONYME, janvier 1779a.

${ }^{13}$ ANONYME, 9 janvier 1779 ; ANONYME 19 janvier.

${ }^{14}$ ANONYME, 20 janvier 1779.

15 ANONYME, 25 janvier, p. 97-9.

${ }^{16}$ ANONYME, 1 février 1779; ANONYME, 15 février 1779.

${ }^{17}$ ANONYME, 2 février 1779.

${ }^{18}$ ANONYME, 2 février 1779, p. 130.

${ }^{19}$ GARAT, 1779.

${ }^{20}$ BRA, 1779.

${ }^{21}$ ANONYME, 15 mars 1782.

${ }^{22}$ ANONYME, novembre 1782.

${ }^{23}$ Trousson, 2006, p. 187.
} 
«si le fond du livre est beaucoup plus riche [que celui de l'Essai-78], la forme en est aussi plus décousue. Il faut prendre son parti de voir l'auteur passer tout à coup du palais de César au grenier de MM. Royou ${ }^{24}$, Grosier ${ }^{25}$ et consorts, de Paris à Rome, de Rome à Paris, du règne de Claude à celui de Louis XV, du collège de la Sorbonne à celui des augures, s'adresser tantôt aux maîtres du monde, tantôt aux derniers roquets de la littérature, et dans son enthousiasme dramatique, faire parler les uns, répondre les autres, s'apostropher lui-même, apostropher ses lecteurs et leur laisser souvent l'embarras de chercher quel est le personnage qu'il fait parler, ou quel est celui auquel il s'adresse $\mathrm{s}^{26} \gg$.

De plus, là où dans l'Essai-78 la trame narrative était interrompue par de nombreuses notes en bas de page, dans l'Essai-82 le lecteur se perd dans un système double, fait de notes qui sont toutes en fin de chaque volume, mais les unes numérotées et les autres composées ou d'un chiffre ou d'une lettre, ou des deux ${ }^{27}$. Les premiers éditeurs, Jules Assézat et Maurice Tourneux, déclarèrent en 1875 : «La plume une fois reprise, [Diderot] ne sut pas la déposer à temps, et c'est ce qui donne à cette seconde édition une physionomie indécise et troublée ${ }^{28} \gg$. Et pourtant, l'Essai-82 n'est, me semble-t-il, ni plus difficile à suivre qu'un roman tel que Jacques le fataliste, ni plus déroutant à lire qu'un article du Dictionnaire de Pierre Bayle. Ce qui gêne n'est pas sa dimension esthétique, mais celle, pour ainsi dire, politique et éthique. Comme on va le voir, le texte-querelle risquait de compromettre la réputation du philosophe au moment même où il importait de la soigner pour la postérité, et il semble encore représenter un risque. Mais quel était donc - quel est encore - le sujet de la querelle ? Quelle est son importance si le fait même de quereller peut avoir des enjeux politiques et éthiques ?

\section{Sujet de querelle/sujet à querelle}

Nous l'avons souvent constaté dans le projet AGON : une querelle peut en cacher une autre. L'Essai dans ses deux formes présente un cas intéressant dans la mesure où l'on a affaire à plusieurs querelles qui se superposent et qui s'enchaînent dans le temps.

\section{La querelle des philosophes}

D'abord, il y a le simple effet de « chiffon rouge » de toute publication de Diderot. L'Essai78 était le premier ouvrage publié par Diderot depuis presque quinze ans, le premier depuis vingt ans qui se publiait avec privilège. Rappelons que l'Encyclopédie avait été privée de privilège en 1759 dans un climat de querelles très vives, dont le point culminant fut, sans doute, la représentation sur la scène de la Comédie française en 1760 de la comédie de Charles Palissot, Les Philosophes, qui offre non seulement une critique générale des idées philosophiques, mais aussi une satire personnelle d'Helvétius, de Diderot et de Rousseau, ce dernier se trouvant représenté à quatre pattes et mangeant une feuille de laitue ${ }^{29}$. Rappelons aussi que les derniers volumes de l'Encyclopédie étaient sortis dans la clandestinité en 1765,

\footnotetext{
${ }^{24}$ Thomas Marie Royou, journaliste et frère de Mme Fréron, était peut-être responsable des comptes rendus de l'Essai-78 dans l'Année littéraire en 1779.

${ }^{25}$ Jean-Baptiste Grosier fut l'éditeur du Journal de littérature, des sciences et des arts.

${ }^{26}$ GRIMM, 1880, p. 104.

${ }^{27}$ Pour l'explication de ce système, voir DIDEROT, 1782, t. I, p. 282.

${ }^{28}$ DIDEROT, 1875-77, t. 3, p. 6.

${ }^{29}$ Pour une étude sur la constitution de ce camp, voir MASSEAU, 2000, et FERRET, 2007.
} 
et que Diderot a écrit les textes pour lesquels il est connu aujourd'hui - les Salons (17591781), le Rêve de d'Alembert (1769), Jacques le fataliste (1778-80) - pour la Correspondance littéraire, journal manuscrit à circulation très limitée. Ainsi, même vingt ans après la comédie des Philosophes, une publication de Diderot, quel que soit le sujet traité, était sujette à querelle.

Son nom a beau ne figurer nulle part dans l'Essai, il se devine et se devinait facilement à partir de la préface dédiée à «Monsieur $\mathrm{N}^{* * * 30}$ » (Naigeon) et qui fait allusion au « $\mathrm{B}$. $\mathrm{d}^{\prime} \mathrm{H}^{31} »$ (Baron d'Holbach), tous les deux connus pour être des amis proches de Diderot. Ce pseudo-anonymat a attiré l'attention de la critique : à la fin d'une notice qui insiste de manière audiblement ironique sur l'anonymat de l'auteur, le Journal de littérature, des sciences et des arts fait allusion au Fils naturel (1757), comédie de Diderot : " Nous avons poussé la délicatesse jusqu'à ne pas nommer le père de ce fils naturel et illégitime, quoique tous les échos et un bout d'oreille le désignent assez clairement ${ }^{32} \gg$. Ce dévoilement enjoué de l'identité de l'auteur est d'autant plus significatif qu'il installe l'Essai-78 dans ce qu'on pourrait appeler «une perspective de querelle perpétuelle » car non seulement Le Fils naturel avait été lui aussi l'objet d'une querelle (suite à une accusation de plagiat ${ }^{33}$ à laquelle le journaliste fait évidemment aussi allusion), mais la querelle de la paternité du Fils faisait partie d'une querelle beaucoup plus ample et qui battait son plein dans la deuxième moitié des années 50, à savoir, bien sûr, celle de l'Encyclopédie, autre enfant controversé de Diderot. Étant le continuateur du Journal de Trévoux - rappelons le rôle majeur joué par les jésuites dans la querelle de l'Encyclopédie, ouvrage qui concurrençait le Dictionnaire de Trévoux - le Journal de littérature, des sciences et des arts ne pouvait pas ne pas réagir. Il en va de même des autres journaux ; jésuites ou non, ils reprennent les termes de la querelle de l'Encyclopédie, connu aussi sous le nom de "l'affaire des Cacouacs », ce dernier terme faisant référence à cette race à part qu'étaient les philosophes selon leurs adversaires : les Affiches, annonces et avis divers de l'abbé de Fontenai accusent l'auteur de l'Essai-78 d'être « un déclamateur obscur et néologique ${ }^{34}$ », termes qui rappellent les reproches faits à un autre ouvrage de Diderot, De l'interprétation de la nature (1754), par Jacob Nicolas Moreau dans son Nouveau Mémoire sur les Cacouacs $(1757)^{35}$; et dans sa lettre au Journal de Paris, Garat reproche à l'auteur du compte rendu de l'Essai-78 d'avoir simplement " pris le parti de trouver le même défaut dans tous les ouvrages du même auteur ${ }^{36}{ }^{3}$. L'Essai-78, on le voit bien, fait resurgir des réflexes de partis déjà constitués lors d'une querelle antérieure.

Ces réflexes partisans, Diderot comptait-il dessus, jouait-il même à les provoquer ? On y reviendra plus tard. Pour l'instant, passons à la question suivante : que l'Essai-78 soit ou non un chiffon rouge agité par Diderot de façon provocatrice, qu'en est-il de ce que dit l'ouvrage des règnes de Claude et de Néron, de la vie et des écrits de Sénèque ? Cela ne compte-t-il pas, ou fort peu, dans cette querelle?

Le « cas Sénèque », qui se transforme en querelle

\footnotetext{
${ }^{30}$ DiDEROT, 1778 , p. 1. Le nom apparaît dans DiDEROT, 1782, t. 1, p. 1.

${ }^{31}$ Diderot, 1778, p. 8. Le nom apparaît dans Diderot, 1782, t. 1, p. 5.

${ }^{32}$ ANONYME, 1779a, p. 372.

33 Voir Tunstall, 2002. Conroy observe dans son compte rendu : « Tunstall collects 25 contemporary documents that recreate the « ambiance critique » in the warring camps and cabals that either panned or praised Le Fils. For me, the winners of this polemic are Diderot's enemies » (CONROY, 2003, p. 205).

${ }^{34}$ ANONYME, 20 janvier 1779, p. 10 (colonne de gauche).

${ }^{35}$ Voir STENGER 2004.

${ }^{36}$ GARAT, 1779, p. 177.
} 
Malgré ce qu'en dit Garat, la presse a lu l'Essai-78 de près et discute dans le détail sa présentation des règnes de Claude et de Néron, et surtout sa présentation de Sénèque, de sa vie et de ses écrits. En fait, c'est un vieux débat ${ }^{37}$ dans l'historiographie que le personnage de Sénèque, instituteur et conseiller du monstre, Néron, et les écrits ne sont pas moins controversés que le personnage depuis aussi loin que la diatribe de Quintilien dans les Institutio oratoria ${ }^{38}$, texte capital dans l'histoire de l'enseignement de la rhétorique en France.

Ce que l'on peut appeler «le cas Sénèque » se structure autour de trois questions : premièrement, y a-t-il contradiction ou non entre la conduite de Sénèque et sa philosophie ; et si oui, que s'ensuit ? C'est-à-dire, quand le philosophe romain conseillait la vertu, la pratiquait-il lui-même ? On discute de la fortune immense du stoïcien, de la manière dont il l'a amassée et de l'usage qu'il en a fait; de la présence à la cour d'un auteur d'un ouvrage sur la retraite ; et surtout du rôle joué à la cour du tyran meurtrier par un auteur d'un ouvrage sur la clémence. Deuxièmement, que penser de la philosophie stoïcienne ? L'idée selon laquelle la liberté et le bonheur sont à rechercher dans la retraite du monde et dans le calme des passions, est-elle bonne ? Est-elle praticable ? Et troisièmement, y a-t-il contradiction ou non entre la philosophie de Sénèque, qu'elle soit bonne ou mauvaise, et le style dans lequel elle s'exprime ? Son goût du jeu de mot, par exemple, est-il compatible avec le sérieux philosophique, ou est-ce le signe d'un bel-esprit qui ne cherche qu'à briller ? Il y va donc de sa vie, de sa philosophie et de son écriture.

Dans l'Essai-78, Diderot entreprend une relecture de toutes les sources et de tous les points de vue de Tacite à Batteux en passant par Sullius, Quintillien, Michel de Montaigne, François de la Rochefoucauld, Charles de Saint-Évremond et Julien Offray de La Mettrie (entre autres), en vue de défendre Sénèque contre les accusations de mauvais écrivain, mauvais philosophe et mauvais homme, de «faire pour l'innocence du philosophe, ce que vous [Naigeon et Nicolas La Grange, traducteurs et éditeurs] avez fait pour l'intelligence de ses écrits ${ }^{39}$. La critique répond, comme elle se doit, en accusant Diderot de justifier ce qui est, selon elle, cupidité et hypocrisie de la part de Sénèque et, pire encore, sa lâcheté devant Néron, complicité avec le meurtre ; elle l'accuse aussi d'approuver un style d'écriture qui tient du mauvais goût, et de souscrire au stoïcisme, dont la morale douteuse ne se fonde sur aucune référence surnaturelle.

Ces arguments n'ont rien de très nouveau en eux-mêmes ${ }^{40}$. Cependant, si le cas Sénèque est réouvert en 1778, si le vieux débat se réchauffe et se rejoue sous forme de querelle, c'est en grande partie parce que, les accusations faites à Sénèque et réfutées par l'essayiste ressemblant à celles faites à Diderot lui-même et aux autres philosophes par les anti-philosophes, l'apologie du philosophe antique par le philosophe moderne est susceptible d'être lue comme une auto-apologie, une apologie de lui-même et des philosophes. N'oublions pas que la question se posait depuis quelque temps de la relation que devaient avoir les philosophes et les hommes de lettres avec ceux que Jean Le Rond d'Alembert appelaient les « grands »dans son Essai sur la société des gens de lettres et des grands $(1753)^{41}$; que Voltaire était depuis longtemps identifié avec la cour prussienne, y ayant fait au tout début des années 50 un séjour de trois ans (plein de querelles d'ailleurs); et que si, à la fin des années 50, Diderot n'avait pas accepté l'offre de Catherine II de faire publier l'Encyclopédie en Russie après son interdiction en France, il accepta en 1773 son invitation à venir à la cour de Saint-Pétersbourg et passa cinq mois chez l'impératrice (d'octobre 1773

\footnotetext{
${ }^{37}$ William Conroy parle de « querelle », voir CONROY, 1975 ; Masseau, d'une polémique, voir MASSEAU, 2014.

${ }^{38}$ QUINTILLIEN, Institutio oratoria, 10.1.125-31.

${ }^{39}$ DIDEROT, 1778 , p. 8.

${ }^{40}$ Voir CONROY, 1975.

${ }^{41}$ D'AlEMBERT,: D’ALEMBERT, 1753, p. 83-163.
} 
jusqu'en mars 1774). Dans le compte rendu, les Annonces, affiches et avis divers posaient une série de questions, les unes plus ironiques que les autres :

«Depuis quelque temps il est fort question de ce Sénèque. Nos philosophes s'agitent, se tourmentent pour célébrer ses mœurs, ses talents, et pour le défendre envers et contre tous. Mais quel rapport peut-il y avoir entre eux et lui ? Le regarderaient-ils comme un de leurs héros ? Sa doctrine serait-elle conforme à la leur ? Le reproche d'avoir corrompu le goût de leur siècle retomberait-il sur eux comme sur lui ? Ses actions auraient-elles démenti son frivole étalage de belles maximes ? Ce rhéteur moraliste, n'aurait-il été regardé, même de son temps, que comme un misérable charlatan de vertu, un hypocrite raffiné, un vil adulateur, un lâche complaisant de la débauche et des extravagances de Néron, un homme dévoré de la soif des richesses $?^{42}{ }$.

Mais l'ironie est d'autant plus grande que Diderot joue précisément sur l'identification entre Sénèque et les philosophes modernes. Avant d'examiner les mécanismes textuels de cette identification, observons que si le cas de Sénèque se transforme en querelle de Sénèque pour prolonger ou se superposer à la querelle des philosophes, les camps des «philosophes » et des « anti-philosophes », formés dans les années 1750, ne sont plus tout à fait les mêmes en 1778 , et qu'une des grandes différences concerne Rousseau, autre sujet de - et sujet à querelle.

\section{Une querelle de philosophes}

Si « le citoyen de Genève »s'était vu satirisé au même titre que Diderot et Claude-Adrien Helvétius dans la comédie de Palissot, il avait déjà, pour sa part, affiché sa rupture avec les encyclopédistes, notamment dans sa Lettre à d'Alembert sur les spectacles (1758), qui répondait à l'article "Genève ». Cet article était très critique à l'égard de la ville natale de Rousseau, à cause de son hostilité à l'égard du théâtre, art que la tradition calviniste proscrivait depuis 1617, mais qui était, depuis les troubles des années 30 et la présence de la France comme pouvoir médiateur, identifié à la culture française. La proscription religieuse était ainsi transformée en arme contre ce qui pouvait être perçu comme un "impérialisme » culturel $^{43}$. La présence, aux portes de Genève, de Voltaire, le plus grand tragédien français et qui avait participé à l'article signé d'Alembert, n'aidait évidemment en rien à cette situation ; et le public voyait par la suite une querelle quasi continue au long des années 60 et 70 entre le sage de Ferney et le citoyen de Genève. Diderot avait pris la décision de ne pas y participer et de garder le silence sur son désaccord avec celui qui avait été son $\mathrm{ami}^{44}$. Rappellons que dans les années 40, Rousseau et Diderot avaient eu, avec Étienne Bonnot de Condillac, le projet de fonder ensemble un journal sous le titre du Persifleur, et que Rousseau était venu voir Diderot lors de son séjour en prison en 1749 , mais que leur relation commença à être plus tendue au moment où Rousseau refusa la pension royale qui lui avait été offerte après la représentation du Devin du village (1752) - Diderot lui avait conseillé de l'accepter, provoquant ce que Rousseau appellera plus tard « une querelle très vive ${ }^{45}$ ». La relation finit par se rompre en 1757, à la suite de deux phrases prononcées dans Le Fils naturel de Diderot, à savoir

\footnotetext{
${ }^{42}$ ANONYME, 20 janvier 1779, p. 10 (colonne de droite).

${ }^{43}$ MARKOVITS, 2009.

${ }^{44}$ Voir CitTON, 2004.

${ }^{45}$ Rousseau, 1995, p. 464.
} 
«l'homme de bien est dans la société » et « il n'y a que le méchant qui soit seul ${ }^{46}$ : non seulement Rousseau s'opposa aux vues énoncées dans ces maximes, mais il s'y voyait attaqué $^{47}$. Or, si l'on a pu identifier un certain nombre d'allusions possibles à Rousseau, sans qu'il soit nommé, dans une série d'articles de l'Encyclopédie écrits par Diderot (dont « Haine », « Innocence », «Indigne ${ }^{48}$ ), l'encyclopédiste ne répondait pas de manière directe, explicite ou personnelle. Il écrit à Êtienne Maurice Falconet à propos de son ancien ami dans une lettre du 6 septembre 1769 : « quelque chose qu'il invente, qu'il controuve, qu'il dise, qu'il fasse, je ne donnerai jamais au public le scandaleux spectacle de deux amis qui se déchirent ; que je me respecterai moi-même ; que je respecterai d'honnêtes gens qui me sont chers et que ma défense compromettrait ${ }^{49} \gg$.

Et pourtant, en 1778, l'année où meurent et Voltaire et Rousseau, Diderot semble revenir sur sa décision et prendre la parole. Dans une note en bas de page de l'Essai-78, son essayiste enjoint au lecteur de : " déteste[r] l'ingrat qui dit du mal de ses bienfaiteurs; déteste[r] l'homme atroce qui ne balance pas à noircir ses anciens amis; déteste[r] le lâche qui laisse sur sa tombe la révélation des secrets qui lui ont été confiés, ou qu'il a surpris de son vivant $»^{50}$. Rousseau n'est pas nommé, mais les lecteurs ont cru reconnaître des références à son refus de pension royale et à ses Confessions qui, sans être imprimées encore, connaissaient depuis 1770 une certain publicité due à la circulation manuscrite de l'ouvrage et, surtout, aux lectures qu'en faisait Rousseau lui-même dans quelques salons parisiens. Devant cette note, la presse crie au scandale ; les Annonces reprochent à Diderot de parler ainsi d'un " homme qui a honoré ce siècle par ses talents » et s'étonnent de ce que le philosophe vienne " ensuite nous prêcher la douceur, l'humanité, la bienfaisance, la tolérance ! ${ }^{51} »$. Et pourtant, dans l'Essai-82, l'essayiste réitère ses reproches et incorpore non seulement ce qui était une note en bas de page dans le corps même du texte, mais il attire l'attention du lecteur, en disant qu'elle avait fait « un grand bruit ${ }^{52} »$, et il y supplée ${ }^{53}$.

\section{Gêne critique et méta-querelle}

Il est donc aisé de comprendre pourquoi ce texte a pu être exclu du canon littéraire et même marginalisé dans le corpus diderotien : d'une esthétique complexe, il est aussi d'une politique et d'une éthique apparemment douteuses, compromettant les valeurs des Lumières, la réputation des philosophes, et du philosophe que fut Diderot, à qui il ne sied pas de quereller. Qu'un philosophe réponde aux attaques prévisibles des journalistes depuis longtemps hostiles est déjà quelque peu problématique dans la mesure où il semblerait ou qu'il élève au rang d'adversaires légitimes des figures considérées par les études littéraires de nos jours comme très mineures, et par les historiographes de la République française comme ayant eu tort, ou qu'il s'abaisse à leur niveau. Et pourtant, se quereller avec des journalistes est moins grave que de se quereller avec un autre philosophe. Que deux philosophes pratiquent la disputatio ou la controversia dans le but de chercher la vérité, c'est une chose, mais qu'ils pratiquent la querelle, dont l'horizon n'est pas la vérité mais l'intérêt personnel, et le ton celui de la passion plutôt que celui de la raison, c'est aller à l'encontre de l'ethos philosophique.

\footnotetext{
46 DIDEROT, 1994-99, t. 4, p. 113.

${ }^{47}$ Voir RoUSSEAU, 1965-1995, t. 4, p. 166-7. L'autographe de cette lettre est inconnu.

${ }^{48}$ Voir LECA-TSIOMIS, 1999, p. 459-85.

${ }^{49}$ DIDEROT, 1994-1999, t. 5, p. 848-49.

${ }^{50}$ DIDEROT, 1778, p. 121.

${ }^{51}$ ANONYME, 20 janvier 1779, p. 10 (colonne de droite).

52 DIDEROT, 1782, t. 1, p. 126.

${ }^{53}$ DidEROT, 1782 , t. 1 , p. $126-40$.
} 
Pour expliquer les querelles de Rousseau et de Voltaire, la critique a souvent eu recours à un vocabulaire pathologique : on les a dits «susceptibles », voire, dans le cas de Rousseau, « fou $^{54}$ ». En ce qui concerne Diderot et la publication de l'Essai-78 et -82, les explications à teinture psychologique ne manquent pas, à la fois chez les critiques de l'époque qui l'accusent de folie ou, plutôt, d'enthousiasme ${ }^{55}$, et chez les critiques contemporains, même chez ceux qui affirment ne pas croire à ce genre d'explication ${ }^{56}$. Cependant, elles sont d'autant moins convaincantes que l'essayiste se montre extrêmement attentif, précisément, aux effets esthétiques, politiques et éthiques de son texte-querelle.

Si dans 1'Essai-78, l'essayiste donne à Naigeon le pouvoir de censurer son ouvrage ${ }^{57}$, dans l'Essai-82, il va jusqu'à inclure les conseils qu'il aurait reçus de ses amis suite à la querelle enclenchée par l'Essai-78 :

«La fastidieuse répétition des mêmes imputations entraînera une répétition non moins fastidieuse des mêmes réponses, et il serait facile que vous gâtassiez votre ouvrage en l'allongeant. Votre réplique serait excellente, qu'elle aurait au moins l'inconvénient d'arracher à l'obscurité des ouvrages et des noms faits pour y rester. Demeurez en repos ; épargnez-vous à vous-même le mal que vous vous feriez $^{58} »$.

Impossible donc de dire que Diderot ait publié l'Essai-82, ni même l'Essai-78, sans savoir ce que cette publication pouvait lui valoir. Ainsi, non seulement le texte-querelle est-il un supplément à la querelle qu'avait engendrée la première édition du texte et un supplément de querelle qu'il fait redémarrer : c'est aussi une réflexion sur la querelle elle-même.

Mais pour être de l'ordre du «méta- », cette réflexion ne participe pas moins de la querelle. C'est ce que montre l'impératif servant de titre à mon propos - « Ne nous engageons point dans les querelles »- impératif qui est aussi une préterition, un performatif à l'envers car, en s'énonçant, il accomplit l'action contraire à celle à laquelle il fait référence, c'est-àdire qu'il engage l'énonciateur dans une querelle. L'action performative de l'énoncé a pour effet supplémentaire de faire subir à cet énoncé-même une petite modification : dans l'Essai78, il apparaît sous la forme de «ne nous engageons point dans des querelles ${ }^{59}$ »; quatre ans plus tard, ce partitif quelque peu vague s'est transformé en article défini, les querelles ayant été effectivement engagées par le premier énoncé paradoxal (et elles seront réengagées par le deuxième). Mais comment comprendre cet écart ironique entre le dire et le faire ? Diderot ne se rend-il pas compte de la performativité querelleuse de son dire ? Se distancie-t-il de son personnage d'essayiste qui semble ignorer l'effet querelleur de ses mots ? L'Essai, est-il un texte-querelle malgré son auteur ou malgré son personnage principal d'essayiste ? Regardons de plus près la logique argumentative de ce dernier.

Ceci n'est pas une querelle?

\footnotetext{
${ }^{54}$ Voir TROUSSON, 2000 ; WACJMAN, 1996.

${ }^{55}$ ANONYME, 20 janvier, 1779, p. 11 (colonne de droite).

${ }^{56}$ Il est surprenant de voir, par exemple, Yves Citton commencer une phrase par : "Moins paranoïaque que Rousseau, Diderot... », CITTON, 2004, p. 93. Charles Vincent, pour sa part, parle du « caractère enthousiaste » de Diderot qui fait qu'il « s'emporte violemment», VINCENT, 2014, p. 596-7.

57 «Disposez de mon travail comme il vous plaira ; vous êtes le maître d'approuver, de contredire, d'ajouter, de retrancher » (DIDEROT, 1778, p. 7).

${ }^{58}$ DIDEROT, 1782, t. 1, p. 211.

${ }^{59}$ DIDEROT, 1778, p. 285-6.
} 
L'essayiste joue un certain nombre de rôles dans le texte, à savoir le lecteur de Sénèque, son «juge ${ }^{60} »$, son « avocat ${ }^{61} »$, son « apologiste ${ }^{62} »$, et il joue beaucoup sur le discours de l'amitié, thème sénéquien par excellence. À travers ces rôles, il cherche à persuader son lecteur non seulement de se joindre à lui, lecteur, avocat et ami de Sénèque, mais aussi de se transformer, par le moyen de la lecture de l'Essai, en avocat, en apologiste et en ami de l'essayiste. Sa stratégie de persuasion, dont nous allons étudier le fonctionnement consiste moins à mobiliser une argumentation rationnelle avec des preuves à l'appui, qu'à démontrer le non-rationnel qui sous-tend tous les points de vue, y compris le sien, et à faire appel aux passions et à la vertu, les siennes ainsi que celles du lecteur.

Son appel à la vertu semble avoir eu récemment un certain succès critique, du moins en ce qui concerne ce que l'on pourrait appeler « le cas Diderot », ou plutôt, « le cas du Diderot de l'Essai », succès qui n'est sans doute pas sans rapport avec le succès (même si c'est davantage dans le monde anglo-saxon) de la branche des études philosophiques et littéraires connue sous le nom d'éthique de la vertu ${ }^{63}$, branche qui s'appuie notamment sur les œuvres de Sénèque ${ }^{64}$. Selon une étude des derniers œuvres de Diderot, parue en 2014, le philosophe des Lumières proposerait dans l'Essai un protocole de lecture des œuvres de Sénèque et des histoires des règnes de Claude et de Néron qui constitue " une éthique de l'interprétation ». ${ }^{65}$ L'auteur, Charles Vincent, résume le protocole ainsi : « Le philosophe doit être en premier lieu un bon lecteur, c'est-a-dire un lecteur qui pèse les documents, les témoignages, les preuves, mais aussi un lecteur qui adopte une attitude bienveillante à l'égard de l'univers de signes qu'il est amené à décrypter ${ }^{66} »$; qui plus est, il dit l'appliquer aux textes de Diderot, cherchant, par exemple, à défendre le philosophe contre une lecture féministe de son texte, Sur les femmes $(1772)^{67}$.

Or il est vrai, comme on va le voir, que l'essayiste vante son herméneutique comme vertueuse, se présente comme bon lecteur, comme ami-lecteur, et qu'il essaie de faire adopter par son propre lecteur ce même protocole de lecture. Mais la tâche du critique littéraire consiste à exposer le fonctionnement de la machine textuelle et non pas à la faire fonctionner dans sa propre pratique interprétative de manière à incarner le lecteur idéal projeté par l'essayiste, surtout que, dans le cas précis, ce serait minimiser la dimension " texte-querelle » fondamentale à l'Essai. Il ne sera évidemment pas question ici d'incarner simplement l'autre rôle projeté par le discours de l'essayiste, à savoir le mauvais lecteur ; il s'agit plutôt de démontrer la logique profondément partisane de l'Essai, d'exposer et d'expliquer son attitude hautement et délibérément paradoxale vis-à-vis de la querelle.

\section{Espaces de paix, espaces de conflits}

Dans la préface dédiée à Naigeon, «mon ami ${ }^{68} », l^{\prime}$ 'essayiste fait son autoportrait dans lequel il incarne un certain nombre de vertus associées à Sénèque ou, du moins, à une certaine image du philosophe stoïcien : âgé et vivant dans sa retraite, à l'abri des passions de la jeunesse et des ambitions mondaines, il s'entretient nonchalamment avec des amis, vivants et morts, dont Sénèque lui-même. Devant la question de savoir pourquoi l'essayiste s'emploie à innocenter

\footnotetext{
${ }^{60}$ DiDEROT, 1782, t. 1, p. 236.

${ }^{61}$ Diderot, 1782 , t. 1 , p. 6.

${ }^{62}$ DIDEROT, 1782, t. 1, p. 93.

${ }^{63}$ Voir, par exemple, DENT, 1996.

${ }^{64}$ Voir, par exemple, NUSSBAUM, 1998.

${ }^{65}$ Voir VINCENT, 2014, p. 451-633.

66 Ibid., p. 631.

${ }^{67}$ Ibid., p. 142-51. Voir TunSTALL, 2014.

${ }^{68}$ DIDEROT, 1782 , t. 1 , p. 5.
} 
le philosophe ancien, question que Sénèque pose lui-même, il répond : « tu es et tu seras à jamais [...] un des plus doux liens entre mes amis et moi, entre les hommes instruits de tous les âges, et leurs amis ${ }^{69} »$. Présentant la lecture de son essai comme étant donc un moyen de faire partie de ce groupe transhistorique d'amis, amateurs de Sénèque, l'essayiste dessine une image de son lecteur : d'un âge semblable au sien, s'il lisait Sénèque, une plume à la main, il « referait cet ouvrage à peu près tel qu'il est ${ }^{70}$ », à savoir un texte dans lequel : « je ne compose point, je ne suis point auteur », dit-il, « je lis ou je converse, j'interroge ou je réponds $^{71} »$. Ainsi donc, si écrire un essai sur Sénèque, c'est s'entretenir avec Sénèque, lire l'essai, c'est se joindre à une conversation entre des gens qui s'identifient les uns avec les autres : l'essayiste envisage un lecteur qui aura plus ou moins de profit à lire son ouvrage pour peu qu'il se mette «plus [ou moins] fidèlement à ma place » et qu'il ait « plus ou moins d'analogie avec le philosophe et avec moi ${ }^{72} »$. Ainsi la préface présente-t-elle un espace privé, intime, où les gens, des amis, tombent d'accord soit pour trouver irréprochable la conduite de Sénèque à la cour de Néron, soit pour lui pardonner, si jamais il s'avère impossible de prouver son innocence. Ils se ressemblent non seulement dans leur admiration des préceptes qui se trouvent dans les écrits de Sénèque, mais aussi dans leur tentative de les mettre en pratique :

«au défaut du succès [du projet d'innocenter Sénèque], on ne nous ravira point à vous, à moi, et à quelques autres écrivains qui m'ont précédé dans la même carrière, et dont le travail ne m'a pas été inutile, la gloire de la tentative. À cet avantage tâchons, mon ami, d'en ajouter un second, plus précieux peut-être : qu'il ne vous suffise pas d'avoir éclairci les passages les plus obscurs du philosophe ; qu'il ne me suffise pas d'avoir lu ses ouvrages, reconnu la pureté de ses mœurs, et médité les principes de sa philosophie: prouvons que nous avons su, l'un et l'autre, profiter de ses conseils. Si nous interrogions Sénèque, et qu'il pût nous répondre, il nous dirait : «Voilà la vraie manière de louer mes écrits, et d'honorer ma mémoire ${ }^{73} »$.

C'est un espace d'amitié, de sympathie, de consensus.

Il s'oppose à l'espace dans lequel l'essayiste présente Sénèque, à savoir la cour de Claude et puis celle de Néron, espace des ambitions mondaines et des passions. L'essayiste explique :

«J'aurais pu ne recueillir des règnes de Claude et de Néron que les endroits où Sénèque est en action, et ne montrer que cette grande figure isolée ; mais il m'a semblé que, placé au centre du tableau, on sentirait plus fortement la difficulté et la dignité de son rôle : le gladiateur antique serait plus intéressant, s'il avait en face son antagoniste. D'ailleurs cette manière s'accommodait mieux avec ma nonchalance. Quand on ne présente sur la toile qu'un seul personnage, il faut le peindre avec la vérité, la force et la couleur de Van Dyck; et qui est-ce qui sait faire un Van Dyck $?^{74}{ }^{»}$.

S'étant présenté comme entouré dans sa retraite d'amis, véritables et imaginaires, l'essayiste explique qu'il a choisi de présenter Sénèque plutôt au cœur de l'arène, face à un adversaire,

\footnotetext{
${ }^{69}$ DIDEROT, 1782 , t. 1 , p. 8.

${ }^{70}$ DIDEROT, 1782 , t. 1 , p. 3.

${ }^{71}$ DIDEROT, 1782 , t. 1 , p. 3.

${ }^{72}$ DIDEROT, 1782 , t. 1 , p. 4.

${ }^{73}$ DIDEROT, 1782, t. 1, p. 9-10.

${ }^{74}$ Diderot, 1782, t. 1, p. 4.
} 
présentation qu'il dit ne pas lui avoir coûté d'effort - il se dit «nonchalant » - contraste supplémentaire avec Sénèque qui, en position de combat, ne peut manquer de chaleur. L'espace de la cour, on le comprend, est un espace d'ennemis, de querelles, de dissensus. Deux espaces donc, et deux Sénèque.

Au cours de l'Essai, l'essayiste va changer d'espace, et il sera de plus en plus difficile de le reconnaître dans son autoportrait sénéquien de départ. Le changement se voit de manière assez claire dans un court passage qui se trouvait au milieu de l'Essai-78 mais qui, offrant encore une instance de réflexivité, se voit promu dans l'Essai-82 au statut de préface au deuxième tome. L'essayiste, s'adressant de nouveau à son ami Naigeon, ne s'imagine pourtant plus dans un espace privé, amical ; il se décrit plutôt en public, en terrain hostile, où l'identification avec Sénèque s'avère une sorte de calcul stratégique devant des lecteurs envisagés non pas comme des amis, mais comme des ennemis : «ici [je] présent[e] au censeur » dit-il « le philosophe derrière lequel je me serais caché ; là [je] fais[...] le rôle contraire, et $\mathrm{m}$ 'offr[ $[\mathrm{e}]$ à des flèches qui ne blesseront que Sénèque caché derrière moi $^{75}{ } »$. En fait, il s'agit moins d'identification de sa part que de protection, qui n'est plus, d'ailleurs, réciproque $^{76}$ : qu'il se cache derrière Sénéque, prenne le masque de Sénèque, ou qu'il avance à visage découvert, suivi de Sénèque, l'essayiste imagine réussir toujours à échapper aux attaques qu'il prévoit venir des censeurs. Sénèque l'aurait rendu en quelque sorte invincible dans cette image assez difficile à décrypter ; mais est-ce à condition de le sacrifier ?

Il est vrai qu'à plusieurs reprises, l'essayiste prend ses distances par rapport aux préceptes de Sénèque ; il avait pourtant annoncé dans la préface qu'il allait les suivre : «je ne doute point » dit-il « qu'on ne fît une excellente apologie de Sénèque contre son apologiste, et j'aurais certainement grand plaisir à la lire ; car je désire aussi sincèrement d'avoir tort quand j'attaque, que d'avoir raison quand je défends ${ }^{77} \gg$. Deux de ces préceptes sont importants pour notre propos, à savoir la retraite du monde et le contrôle des passions, c'est-à-dire le mérite ou non de s'engager dans des querelles. Mais avant de regarder les flèches que l'essayiste envoie lui-même à Sénèque, analysons la logique de son apologie.

\section{«Ma façon de lire l’histoire »}

Si Charles Vincent semble avoir été persuadé par la logique discursive de l'Essai, c'est sans doute parce qu'elle est d'un glissant et d'un coercitif assez remarquables. En effet, si l'essayiste commence par présenter sa tentative d'innocenter Sénèque comme fragile, voire comme vouée à l'échec, accusant par une jolie captatio benevolentiae son talent d'avocat de ne pas être à la hauteur ${ }^{78}$, il adopte par la suite, et parfois à l'intérieur d'une même phrase et en glissant d'une position à l'autre, une variété de positions différentes, voire contradictoires. Voici qu'il affirme de l'innocence du philosophe ${ }^{79}$ et de l'excellence de ses écrits ${ }^{80}$; qu'il conclut qu'il y a conformité entre la conduite du philosophe et ses préceptes ${ }^{81}$; qu'il affirme qu'il n'est ni besoin, pour donner des préceptes, d'être en mesure soi-même de les suivre ${ }^{82}$, ni

\footnotetext{
${ }^{75}$ DIDEROT, 1778, p. 273 ; DIDEROT, 1782, t. 2, p. 2.

${ }^{76}$ Sur ce point, je ne suis pas d'accord avec Joanna Stalnaker, qui y voit précisément une relation réciproque : « he can sometimes be found hiding behind Seneca, and other times shielding him from attack » (STALNAKER, 2009, p. 52).

${ }^{77}$ DIDEROT, 1782 , t. 2, p. 41.

${ }^{78}$ DIDEROT, 1782 , t. 1 , p. 6.

${ }^{79}$ DiDEROT, 1782, t. 1, p. 45.

${ }^{80}$ DIDEROT, 1782, t. 2, p. 95.

${ }^{81}$ DiDEROT, 1782, t. 2, p. 71.

${ }^{82}$ DiDEROT, 1782 , t. 1 , p. 86.
} 
besoin, pour les suivre, d'en avoir des exemples ${ }^{83}$; qu'il juge que dans des moments où Sénèque n'a pas obéi à l'un de ses propres préceptes, c'était parce que celui-ci était mauvais ${ }^{84}$; que si Sénèque n'a pas toujours un style pur, il ne faut rien en conclure pour la pureté de ses mœurs ${ }^{85}$; qu'il déclare que l'on ne peut déduire du fait qu'un philosophe écrit bien sur la vertu que sa propre conduite ait été vertueuse ${ }^{86}$; qu'un écrivain ne mérite pas d'être lu, même s'il écrit aussi bien qu'Homère, si sa conduite a été vicieuse ${ }^{87}$; qu'il affirme la possibilité des jugements universels ${ }^{88}$; la nécessité de relativisme dans les jugements de morale $^{89}$; la diversité inévitable dans les jugements ${ }^{90}$; qu'il envisage la possibilité que Sénèque fût coupable ${ }^{91}$; et qu'il présente l'impératif moral de témoigner l'indulgence à son égard ${ }^{92}$.

Le point capital pour notre propos dans cette série de positions se trouve là où l'essayiste offre une réponse à la question suivante : "Comment une assertion a-t-elle pour un critique le caractère de l'évidence, et l'assertion contradictoire a-t-elle également le caractère de l'évidence pour un autre $?^{93} »$. Cette question, loin d'être une sorte de que sais-je ? sceptique, est susceptible de recevoir une réponse, mais celle-ci concerne non pas le caractère de l'évidence, mais le caractère - moral - du critique. L'essayiste s'explique ainsi :

«J'ai ma façon de lire l'histoire. M'offre-t-elle le récit de quelque fait qui déshonore l'humanité ? Je l'examine avec la sévérité la plus rigoureuse ; tout ce que je puis avoir de sagacité, je l'emploie à découvrir quelques contradictions qui me le rendent suspect. Il n'en est pas ainsi lorsqu'une action est belle, noble, grande. Je ne m'avise jamais de disputer contre le plaisir que je ressens à partager le nom d'homme avec celui qui l'a faite. Je dirai plus ; il est selon mon cœur, et peut-être est-il encore selon la justice, de hasarder une opinion qui tende à blanchir un personnage illustre, contre des autorités qui contredisent la teneur de sa vie, de sa doctrine, et l'estime générale dont il a joui. Je me fais honneur d'un pyrrhonisme qu'il est facile d'attaquer, mais qu'il ne serait pas trop honnête de blâmer ${ }^{94} »$.

L'intérêt de ce passage réside non seulement dans le fait que l'essayiste fait glisser l'interrogation de questions épistémologiques à des questions morales, mais aussi dans le fait qu'il y distingue différentes sortes de disputes : celles qu'il pratique, même si le verbe qu'il emploie n'est pas « disputer » mais « examine[r] avec la sévérité la plus rigoureuse »; celles qu'il dit ne jamais pratiquer - « je ne m'avise jamais de disputer contre »; et celles dont il dit qu'elles sont pratiquées par les autres - les deux verbes employés dans ce cas étant «contredire » et " attaquer ». Les deux premières relèvent, selon l'essayiste, de ce qu'il appelle le «pyrrhonisme », terme employé de manière quelque peu surprenante ici dans la mesure où, selon l'usage habituel suivant lequel l'attitude pyrrhoniste consiste à assujettir tout document à un examen critique rigoureux, il devrait ne s'appliquer qu'à la première sorte de

\footnotetext{
${ }^{83}$ Diderot, 1782 , t. 1 , p. 240.

${ }^{84}$ DIDEROT, 1782 , t. 2 , p. $90-1$.

${ }^{85}$ DIDEROT, 1782 , t. 2 , p. 20

${ }^{86}$ DIDEROT, 1782 , t. 1 , p. $227-8$

${ }^{87}$ DIDEROT, 1782, t. 1, p. 198-99. Il s'agit de Lucain.

${ }^{88}$ DIDEROT, 1782 , t. 1 , p. $179-80$.

${ }^{89}$ DIDEROT, 1782 , t. 1, p. 93 , p. $97-100$, p. 107-8.

${ }^{90}$ DiDEROT, 1782, t. 2, p. 186.

${ }^{91}$ DIDEROT, 1782 , t. 2 , p. 45.

${ }^{92}$ DiDEROT, 1782 , t. 1 , p. 45-6, p. 251-6.

${ }^{93}$ DiDEROT, 1782, t. 2, p. 186.

${ }^{94}$ Diderot, 1782 , t. 1 , p. 72.
} 
dispute évoquée, c'est-à-dire à l'examen sévère d'un récit, et non pas au fait de « hasarder une opinion », même si c'est contre les autorités. Qui plus est, dans les deux cas, ce qui provoque soit l'examen critique soit l'envie de hasarder une opinion, n'est pas la vraisemblance ou non du récit, mais le simple plaisir que prend l'essayiste à la lecture et à la propagation d'un récit d'une action exemplaire, plaisir qu'il ne veut pas qu'on lui ôte. L'essayiste se dit ailleurs et à plusieurs reprises tout à fait prêt à être dupe en ce qui concerne l'histoire des grands hommes :

«D'honneur j'ai cru bêtement avec des hommes célèbres, anciens et modernes, que Sénèque était un grand penseur, un instituteur vertueux, un grand ministre ; et si malgré toutes les peines qu[e les censeurs] se sont données pour me détromper, je leur protestais que je persiste dans ma bêtise, ce serait encore de la meilleure foi du monde ${ }^{95} »$.

Il va jusqu'à admirer ceux qui sont prêts à se duper : " Que le petit nombre de ceux qui se tourmentent, qui même s'en imposent, pour trouver des excuses aux grands hommes, est rare, et qu'ils me sont chers $!^{96} »$. Ainsi ce n'est pas que l'essayiste ait raison en ce qui concerne l'innocence de Sénèque ; c'est qu'il est ou qu'il joue à être suffisamment innocent lui-même pour y croire.

La troisième sorte de dispute, celle que pratiquent les autres, ressemble à celle que pratique l'essayiste dans la mesure où il s'agit de porter un regard critique et de contredire un point de vue généralement accepté, sauf que l'objet de la critique, à savoir l'historiographie des hommes illustres, n'est pas le même. C'est ici que l'on voit clairement la nature partisane du texte : l'essayiste, ne prenant aucun plaisir à l'iconoclasme, voit dans la mise en doute des récits des actions des grands hommes non seulement une attaque gratuite, mais aussi un refus de croire simplement à la possibilité que les hommes puissent être à la fois grands et innocents, c'est-à-dire un refus de suspendre tout doute concernant le véridique du récit glorieux. À son sens donc, ce sont plutôt des anti-pyrrhonistes. Et leur refus est présenté sous une lumière morale : se faisant « honneur » et de ne disputer que le récit de quelque fait qui "déshonore » l'humanité, et de recevoir sans examen rigoureux le récit de quelque fait qui l'honore, l'essayiste fait comprendre que, si bien fondé que puisse être d'un point de vue rationnel leur refus de suspendre le doute, il manque d'honnêteté. Si l'essayiste risque d'être bête, ils se déshonorent, eux, lorsqu'ils ne croient ni à la vertu de Sénèque ni à la vertu d'y croire.

Et ils n'ont pas cette foi, selon l'essayiste, parce qu'ils sont excessivement attachés à une certitude historique toujours hors de portée, ou alors parce qu'ils affichent un excès de rigorisme moral — un vice — qui les empêche de se mettre à la place de Sénèque, acte d'identification que l'on a déjà rencontré dans la préface et que l'essayiste décrit comme étant naturel et même chrétien : «Un des beaux préceptes de la morale naturelle et évangélique, c'est de se mettre à la place de l'accusé : que le plus innocent d'entre vous jette la première pierre $^{97} \gg$. Naturelle et chrétienne, cette identification qui permet de comprendre et de ne pas juger $^{98}$, n'est surtout pas stoïcienne et, autre glissement, l'essayiste présente les censeurs de Sénèque comme des adhérents à la morale que l'on associe avec le philosophe, qui pourtant n'y adhérait pas :

«Sénèque s'exhorte à l'examen des choses, sans partialité, sans cette haine

\footnotetext{
${ }^{95}$ DIDEROT, 1782, t. 2, p. 234.

${ }^{96}$ DIDEROT, 1782 , t. 1 , p. 298.

${ }^{97}$ DIDEROT, 1782, t. 2, p. 34.

${ }^{98}$ La phrase «se mettre à la place de » apparait fréquemment, voir DIDEROT, 1782, t. 1, p. 84, p. 89, et t. 2, p. 34, p. 186.
} 
implacable que sa secte a vouée à toutes les autres. D'où venait cette intolérance des stoïciens? De la même source que celle des dévots outrés. Ils ont de l'humeur, parce qu'ils luttent contre la nature, qu'ils se privent et qu'ils souffrent. S'ils voulaient s'interroger sincèrement sur la haine qu'ils portent à ceux qui professent une morale moins austère, ils s'avoueraient qu'elle naît de la jalousie secrète d'un bonheur qu'ils envient, et qu'ils se sont interdit ${ }^{99}{ }^{\gg}$.

Plus loin:

« Je ne crois pas qu'il y eût d'homme moins disposé par caractère à la philosophie stoïcienne que Sénèque, doux, humain, bienfaisant, tendre, compatissant. Il n'était stoïcien que par la tête : aussi à tout moment son cœur l'emporte-t-il hors de l'école de Zenon ${ }^{100}{ } »$.

L'essayiste va jusqu'à dire de la morale stoïcienne, comparable dans sa sévérité la plus rigoureuse à l'examen auquel il soumet le récit de quelque fait qui déshonore l'humanité, non seulement qu'elle est en elle-même un vice mais qu'elle est censée en cacher d'autres : «Censeurs » dit-il «ne vous montrez pas si sévères car je ne vous croirai pas meilleurs ${ }^{101}$ »; "Soyons indulgents, et n'imitons pas les hommes corrompus qui, pour se trouver des semblables, sont de plus cruels accusateurs que les gens de bien ${ }^{102}{ }$. L'essayiste espère ainsi avoir trouvé un moyen de faire taire les censeurs une fois pour toutes : puisque celui qui dispute soit de la vertu de Sénèque, soit du plaisir que prend l'essayiste à la chanter, ne fait qu'à exhiber ses propres défauts, mieux vaut adopter le «tour d'esprit » de l'essayiste car « il convient et à l'homme vertueux qui cherche son semblable, et à l'hypocrite qui cherche une dupe $^{103} »$.

L'essayiste se présente ainsi comme doux, bienfaisant, tendre, compatissant ; ce n'est pas lui qui engagerait une querelle. Et pourtant.

\section{De la colère}

Dans la préface, comme on l'a vu, l'essayiste se présente comme étant dans sa retraite, à l'écart du monde et à l'abri des passions, présentation que l'on reconnaît comme fortement empreinte des valeurs sénéquiennes. L'essayiste examine pourtant ces valeurs de manière critique dans la deuxième partie du texte. Par exemple, les conseils que donne Sénèque à un ami de Lucillus (est-ce Sénèque lui-même?) qui s'est attiré des reproches pour avoir «embrassé le repos, abandonné ses places et préféré l'obscurité de la retraite aux nouveaux honneurs qui l'attendaient », conseils qui consistent à l'exhorter " à se mettre au-dessus de l'opinion : chaque jour il fera sentir à ses censeurs qu'il a choisi le parti le plus avantageux », attirent à Sénèque le reproche suivant de la part de l'essayiste :

«Pour lui, peut-être; mais pour la société ? Il y a dans le stoïcisme un esprit monacal qui me déplaît; c'est cependant une philosophie à porter à la cour, près des grands, dans l'exercice des fonctions publiques, ou c'est une voix perdue qui crie dans le désert. J'aime le sage en évidence, comme l'athlète sur l'arène :

\footnotetext{
${ }^{99}$ DIDEROT, 1782 , t. 2, p. 154-5.

${ }^{100}$ DiDEROT, 1782 , t. 2 , p. 109.

${ }^{101}$ DIDEROT, 1782, t. 1, p. 62.

102 DIDEROT, 1782 , t. 1 , p. 46.

${ }^{103}$ DidEROT, 1782 , t. 1 , p. 104.
} 
l'homme fort ne se reconnaît que dans les occasions où il y a de la force à montrer ${ }^{104} »$.

Que le philosophe doit être actif dans le monde est un point constamment réitéré par l'essayiste contre Sénèque, qu'il accuse d'embrasser des vues «monastiques et antisociales ${ }^{105} »$. Ainsi est-ce précisément le Sénèque de la cour, celui sur qui retombent toutes les accusations d'hypocrisie et de collaboration aux crimes, et non pas le Sénèque de sa retraite que vante l'essayiste. Aussi, tout en se présentant lui-même dans sa retraite, il publie son ouvrage, rentre dans l'espace public. Notons que si, dans l'Essai-78, ces deux actes pouvaient être attribués à deux personnages différents, le premier à l'essayiste et le deuxième à l'auteur Diderot, ce n'est plus le cas dans l'Essai-82, où l'essayiste répond à la réception critique de l'ouvrage de Diderot, comme étant son ouvrage.

En faisant l'éloge du philosophe en action dans le monde et en se présentant lui-même dans le monde par le moyen de la publication de son ouvrage, l'essayiste prend aussi ses distances par rapport à ce que dit Sénèque à propos des passions, et surtout celle de la colère, que l'essayiste présente comme étant un stimulus important pour tout philosophe. Discutant de l'ouvrage de Sénèque sur la colère dans lequel il est dit que « La colère n'est pas conforme à la nature de l'homme ", l'essayiste rétorque : «Je ne connais pas de passion plus conforme à la nature de l'homme. La colère est un effet de l'injure ; et la sagesse de la nature a placé le ressentiment dans le cœur de l'homme, pour suppléer au défaut de la loi »; et en réponse à l'affirmation selon laquelle « La vertu serait bien à plaindre, si la raison avait besoin du secours des vices », il dit :

«C'est que les passions ne sont pas des vices : selon l'usage qu'on en fait, ce sont ou des vices ou des vertus. [...] Je ne conçois pas comment un être sensible peut agir sans passion. Le magistrat juge sans passion ; mais c'est par goût ou par passion qu'il est magistrat ${ }^{106} »$.

Ayant rencontré un passage dans l'ouvrage de Sénèque où le philosophe exhibe de la colère, l'essayiste jubile : «Et cette bassesse, mon philosophe, remplit votre âme de colère, votre bouche d'imprécations! Je vous en loue, mais vous avez oublié vos principes sur la colère. [...] vous sentez juste ; mais, de stoïcien que vous étiez, vous vous êtes fait homme ${ }^{107} \gg$. On l'a déjà vu, l'inhumanité des stoïciens est au cœur de sa critique des censeurs de Sénèque, et ici, comme dans le cas de la compassion, l'essayiste trouve juste l'expression de la colère. L'exprime-t-il dans son texte?

De fait, à plusieurs moments, la nonchalance sénéquienne affichée par l'essayiste dans la préface cède la place à une écriture, pour ainsi dire, «chalante », engagée, passionnelle. Par exemple, Dion Cassius est un « homme pervers, aveuglé par la haine qu'il portait à tous les gens de bien ${ }^{108}$ »; Sullius « un délateur vénal et formidable, un scélérat justement exécré de la multitude des citoyens, un prévaricateur, un concussionnaire ${ }^{109}$, ou encore « un furieux souillé, accusé, puni de mille crimes, un malfaiteur dont le témoignage n'aurait pas été admis au tribunal des lois ${ }^{110} »$. Avouant (ou presque) la violence de ses propos, il glose :

\footnotetext{
${ }^{104}$ DIDEROT, 1782, t. 2, p. 27.

${ }^{105}$ DIDEROT, 1782 , t. 2, p. 55-6.

106 DIDEROT, 1782 , t. 2 , p. 104-5.

${ }^{107}$ DIDEROT, 1782, t. 2, p. 110-11.

${ }^{108}$ DIDEROT, 1782, t. 1, p. 177.

${ }^{109}$ DIDEROT, 1782 , t. 1 , p. 120.

${ }^{110}$ DideroT, 1782 , t. 1 , p. 123-4.
} 
«L'invective invite l'invective. Peut-être me suis-je oublié quelquefois ; mais si cela m'est arrivé, ce ne sera que dans les endroits où la critique s'est déchaînée sans mesure contre des hommes respectables et des talents généralement avoués. Mais alors quel est l'homme assez patient, je dirai même assez ingrat, pour écouter avec une froide indifférence l'insulte adressée à des écrivains qui honorent la nation, et à qui l'on doit les heures de sa vie les plus délicieuses ? Je ne suis pas capable, et fasse le ciel que je meure avant que d'avoir été capable d'une modération que je me reprocherais ${ }^{111} »$.

C'est très clair : la colère est juste lorsqu'elle vise " des agresseurs indécents, malhonnêtes, injurieux, violents ${ }^{112} \gg$. Il est donc de la plus grande importance de construire les ennemis comme étant ceux qui ont commencé ; le cas le plus intéressant de ce point de vue est la fameuse note concernant Rousseau.

L'intérêt est double : il vient de la difficulté à construire Rousseau comme ayant commencé la querelle avant même que ses Confessions soient publiées, et de la série de manœuvres, les unes plus glissantes que les autres, auxquelles il se livre pour justifier son attaque. Dans l'Essai-78, Rousseau n'est pas nommé et la querelle figure à l'état purement hypothétique : l'essayiste parle d' « un homme connu par sa probité et par ses lumières » qui lui aurait demandé d'imaginer s'il " paraissait jamais un ouvrage où d'honnêtes gens fussent impitoyablement déchirés par un artificieux scélérat, qui, pour donner quelque vraisemblance à ses injustes et cruelles imputations, se peindrait lui-même de couleurs odieuses ${ }^{113}$ », personnage dont l'essayiste dit qu'il n'était pas le seul à ne pas croire " qu'il ait existé ou qu'il existe jamais un pareil homme ${ }^{114}$. Vu la manière dont l'essayiste présente Sullius, le lecteur est à même de conclure pour sa part qu'il a déjà existé un homme pareil et qu'il peut donc en exister un autre ; si l'essayiste ne le dit pas, Diderot permet de le faire comprendre. Plus frappante est la manière dont Diderot, après avoir été accusé par la presse d'avoir cherché querelle à Rousseau, fait parler son essayiste dans l'Essai-82 ; celui-ci cite l'accusation du journal et lui répond ainsi :

\section{«'On a dit que ma sortie s'adressait à Jean-Jacques Rousseau'.}

Ce Jean-Jacques, a-t-il fait un ouvrage tel que celui que je désigne ? A-t-il calomnié ses anciens amis ? A-t-il décelé l'ingratitude la plus noire envers ses bienfaiteurs ? A-t-il déposé sur sa tombe la révélation de secrets confiés ou surpris ? Cette lâche et cruelle indiscrétion peut-elle semer le trouble dans des familles unies, et allumer de longues haines entre des gens qui s'aiment? Je dirai, j'écrirai sur son monument: Ce Jean-Jacques que vous voyez fut un pervers. Censeurs, j'en appelle à vous-mêmes, interrogez ceux qui vous entourent ; bons ou méchants, je n'en récuse aucun.

Jean-Jacques n'a-t-il rien fait de pareil ? Ce n'est plus de lui que j'ai parlé.

Existe-t-il, a-t-il jamais existé un méchant assez artificieux pour donner de la consistance aux horreurs qu'il débite d'autrui par les horreurs qu'il confesse de luimême ? J'ai protesté que je n'en croyais rien. Censeurs, à qui donc en voulezvous ? S'il y a quelqu'un à blâmer, c'est vous ; j'ai ébauché une tête hideuse, et vous avez écrit le nom du modèle au-dessous.

Ceux d'entre les gens du monde qui jugent sans partialité, ont dit: Les mémoires secrets dont il est question n'existent-ils pas? La querelle est finie.

\footnotetext{
${ }^{111}$ DIDEROT, 1782 , t. 2, p. 223-4.

${ }^{112}$ Ibid.

${ }^{113}$ DIDEROT, 1778, p. 121.

${ }^{114}$ DIDEROT, 1778, p. 122.
} 
Existent-ils ? Il faut convenir qu'il est fou, qu'il est atroce d'immoler, en mourant, ses amis, ses ennemis pour servir de cortége à son ombre: de sacrifier la reconnaissance, la discrétion, la fidélité, la décence, la tranquillité domestique à la rage orgueilleuse de faire parler de soi clans l'avenir; en un mot, de vouloir entraîner tout son siècle dans son tombeau, pour grossir sa poussière.

Ils ont ajouté : Ce morceau de l'auteur sur Jean-Jacques, si c'est à lui qu'il s'adresse, est violent. Mais que penser d'un homme qui laisse, après sa mort, des Mémoires où certainement plusieurs personnes sont maltraitées, et qui y joint la précaution odieuse de n'en permettre la publicité que quand il n'y sera plus ; lui, pour être attaqué; celui qu'il attaque, pour se défendre? Que Jean-Jacques dédaigne tant qu'il lui plaira le jugement de la postérité, mais qu'il ne suppose pas ce mépris dans les autres ${ }^{115} \gg$.

La «perversité » de ce passage, pour reprendre le mot qu'il utilise pour désigner Rousseau, est très remarquable. L'essayiste blâme la presse d'avoir nommé Rousseau - "Censeurs, à qui donc en voulez-vous ? S'il y a quelqu'un à blâmer, c'est vous ; j'ai ébauché une tête hideuse, et vous avez écrit le nom du modèle au-dessous »; puis, n'ayant pas été le premier à nommer Rousseau, il s'autorise à répéter le nom ${ }^{116}$. Ce faisant, il s'engage dans une querelle posthume car l' «odieux » de la démarche de Rousseau ne se limite pas à «la précaution » de ne publier les Confessions que lorsqu'il ne sera plus et ne pourra plus être attaqué ; il consiste aussi, comme le suggère la phrase qui suit, à ne publier Les Confessions que quand celui que Rousseau y aura attaqué ne sera plus non plus et ne pourra donc plus se défendre. Ainsi l'essayiste se sert de l'accusation faite à son propre égard relative à l'Essai-78, à savoir qu'il a attaqué Rousseau afin de lancer une attaque préventive contre lui, alors nommé, attaque qu'il présente à la fois comme entièrement hypothétique et comme purement défensive. Il se sera déjà défendu contre les attaques quand elles viendront, même après sa mort.

Le fait d'inclure la critique adressée à l'Essai-78 dans l'Essai-82 peut donc se comprendre comme une tentative de justifier la querelle que mène Diderot à l'égard des Confessions de Rousseau, figure que l'essayiste rapproche des adversaires de Sénèque : «Il faut convenir que les ennemis de nos philosophes ressemblent quelquefois merveilleusement aux détracteurs de Sénèque ${ }^{117}$ ». Comme il est clair que ce que cherche Diderot est un moyen d'avoir le dernier mot, un moyen d'avoir déjà eu le dernier mot, examinons, pour conclure, quels sont, précisément, les derniers mots de l'essayiste.

\section{Le dernier mot}

L'essayiste, homme âgé, « voisin du terme où tout s'évanouit ${ }^{118}$ », se déclare très conscient du fait qu'il dira bientôt son dernier mot, sans que ce mot soit le dernier qui sera dit au sujet ni de Sénèque, ni de l'Essai, ni encore de Diderot ; qui plus est, il se présente comme ayant déjà répondu d'avance à la censure de la postérité. De ce point de vue, de tout point de vue, les derniers mots de $l^{\prime}$ Essai-82 sont remarquables :

«Les preuves qui se déduisent des faits sont bornées ; les conjectures du caprice et de la méchanceté sont infinies. On est dispensé de répondre aux objections de la mauvaise foi. J'ai dit: Vous qui troublez dans ses exercices celui qui visite le jour

\footnotetext{
${ }^{115}$ Diderot, 1782, t. 1, p. 126-28.

${ }^{116}$ De la pratique du nom dans la période, voir TUNSTALL et ANDERSON, 2013.

${ }^{117}$ Diderot, 1782, t. 1, p. 256.

${ }^{118}$ DIDEROT, 1782, t. 1, p. 2.
} 
et la nuit les autels d'Apollon, bruyantes cymbales de Dodone, tintez tant qu'il vous plaira, je ne vous entends plus. Si le dernier qui parle est celui qui a raison, censeurs, parlez, et ayez raison ${ }^{119} »$.

La critique littéraire a eu tendance à minimiser l'aggression et l'ironie de ces derniers mots. Selon Joanna Stalnaker, dans un article qui compare l'écriture testamentaire de Diderot avec celle de Rousseau, la dernière phrase de l'Essai-82 serait une «invitation » aux censeurs à juger son ouvrage, et son injonction à avoir raison, « un encouragement implicite à relire (et à relire encore) sa vie et ses écrits avant d'arriver à leur jugement final ${ }^{120} »$. Une telle lecture me semble à la fois affaiblir l'étrangeté de l'injonction, " ayez raison », et réduire à un simple conflit d'interprétations la portée hautement querelleuse des derniers mots, ce qui, dans la perspective d'AGON adoptée ici, ne peut se recevoir. Lu comme un «texte-querelle ", l'Essai prévoit dans ces derniers mots une prolongation à l'infini d'une querelle que l'essayiste annonce - superbement - avoir déjà gagnée. Ayant déjà répondu aux censeurs de l'Essai-78 et s'étant déjà défendu d'avance contre les attaques futures, il énumère les types de censure qui restent - les preuves bornées, les conjectures infinies du caprice et de la méchanceté et les objections de mauvaise foi - en se déclarant non seulement dispensé d'y répondre, mais sourd à leur bruit. Sa surdité est volontaire au moment de la publication de l'Essai-82 qui annonce un refus de publier, disons, un Essai-82 ; mais elle lui sera, il le sait, prochainement imposée par la mort. Et alors, dans un dernier geste, sans doute le plus belliqueux du texte, il enjoint ses censeurs à avoir raison, tout en réduisant ce que ce serait avoir raison au simple fait d'être le dernier qui parle : le plus important, en fait, est d'avoir déjà parlé. Autant dire qu'il a déjà eu raison, et pour toujours.

Pour la postérité, rien de si bon alors ou rien de si mauvais qu'une querelle ? C'est la question que pose lui-même l'essayiste et à laquelle il fournit une réponse paradoxale : il attaque violemment, en se disant défenseur véhément; il avance dans l'espace public, arène de lutte, tout en se présentant comme étant entre amis dans le privé ; il cherche querelle, tout en affirmant que la querelle est ce que pratiquent les autres. Et c'est une question à laquelle l'histoire de la littérature a fourni une réponse très claire dans le fait d'avoir marginalisé l'Essai dans le corpus diderotien ou à tout le moins minimisé sa qualité de « texte-querelle ».

Dans la perspective AGON, pourtant, l'Essai est d'une importance majeure non seulement pour ce qu'il révèle de l'attachement persistant de la part des historiens de la littérature à une vision de la République des Lettres comme un espace irénique ${ }^{121}$, mais aussi pour sa vision de ce qui fonde le consensus et le dissensus : selon l'essayiste, le consensus, du moins en ce qui concerne les mœurs et les écrits des philosophes, ne peut se construire sur des bases épistémiques ; le différend ne peut se régler par une démonstration rationnelle avec preuves à l'appui. La base du consensus, comme celle du dissensus, est de l'ordre du sentimental - c'est une affaire d'identification ou non, de sympathie ou non, d'amitié ou d'inimitié. C'est une logique profondément partisane, voire sectaire - qui non est mecum,

\footnotetext{
${ }^{119}$ DIDEROT, 1782 , t. 2, p. 240-1.

${ }^{120}$ Ma traduction. Stalnaker écrit : «Diderot concludes the Essai by extending an invitation to his reader to judge Seneca, Diderot and the Essai itself. In fact, he even extends this invitation to his censors, whom he attacks throughout the work for their uncharitable attitude toward Seneca and himself. But he accompanies this invitation with the injunction that they be right in their judgement. [...] Earlier in the Essai, Diderot had emphasized that he subjected his own judgements of Seneca to an ulterior re-reading of his works : « S'est-il en effet trompé ? C'est, me disait un ami, ce qu'une seconde lecture m'apprendra ». [...] With his final injunction, he thus implicitly encourages them to go back to his work and life again (and again) before coming to their final judgement », STALNAKER, 2009, p. 54-5. Semblable sur ce point à l'analyse de Stalnaker est celle de Vincent qui prétend que Diderot «propose [dans DIDEROT, 1782] un mode de lecture ouvert », VINCENT, 2014, p. 506.

${ }^{121}$ Le dernier en date étant FUMAROLI, 2015. Pour un compte rendu qui pointe cet attachement, voir RoUDET, 2015 .
} 
contra me est - présentée, qui plus est, comme une affaire de morale, ce qui ne peut que garantir la perpétuité de la querelle. 


\section{LISTE DES REFERENCES}

ANONYME, janvier 1779, "Essai sur la Vie de Sénèque le philosophe », Journal de littérature, des sciences et des arts, p. 177-206.

ANONYME, janvier 1779a, "Essai sur la Vie de Sénèque le philosophe [...] Second extrait », Journal de littérature, des sciences et des arts, p. 343-372.

ANONYME, 9 janvier 1779, «Essai sur la Vie de Sénèque », Année littéraire, p. 36-70.

AnONYME, 19 janvier 1779, "Essai sur la Vie de Sénèque [...] Second extrait », Année littéraire p. 104-136.

ANONYME, 20 janvier 1779, «Essai sur Sénèque », Affiches, annonces et avis divers, p. 1011.

ANONYME, 25 janvier 1779, «Essai sur la Vie de Sénèque le Philosophe », Journal de Paris, p. 97-9.

ANONYME, 1 février 1779, «Essai sur la vie et les écrits de Sénèque », Journal encyclopédique, p. 394-412.

ANONYME, 2 février 1779, «A l'auteur de l'Extrait de l'Essai de la Vie de Sénèque, inséré dans le Journal de Paris du 25 janvier dernier », Journal de Paris, p. 129-130.

ANONYME, 2 février 1779a, «Observations des Auteurs du Journal, sur la lettre précédente », Journal de Paris, p. 130-131.

AnONYMe, 15 février 1779, "Essai sur la vie et les écrits de Sénèque. Dernier extrait », Journal encyclopédique, p. 27-42.

ANONYME, 15 mars 1782, "Essai sur les règnes de Claude et de Néron », Journal encyclopédique, p. 454-67.

ANONYME, novembre 1782, «Essai sur les règnes de Claude et de Néron », Année littéraire, p. 217-251.

ALEMBERT, 1753, Essai sur la société des gens de lettres et des grands, sur la réputation, sur les mécènes, et sur les récompenses littéraires, dans Mélanges de littérature, d'histoire, et de philosophie, Berlin, [sans nom d'éditeur], t. 2, p. 83-163.

Bonnet (Jean-Claude), 1992, «Diderot et la postérité », Cahiers Textuel, 11, p. 125-132.

Bonnet (J.-C.), 1998, Naissance du Panthéon : essai sur le culte des grands homme, Paris, Fayard.

«BRA », 1779, «Lettre au sujet du nouvel Essai sur la Vie de Sénèque », Journal de Paris, 6 avril, p. 383-5.

CABANE (Franck), 2004, « D'un Essai 1'autre, métamorphose d'un texte », Recherches sur Diderot et l'Encyclopédie, 36, Langres, Société Diderot, p. 17-27.

CitTon (Yves), 2004, «Retour sur la misérable querelle Rousseau-Diderot », Recherches sur Diderot et l'Encyclopédie, 36, Langres, Société Diderot, p. 57-95.

CONROY (William), 2003, compte rendu de CRONK, (Nicholas), (éd), 2002, Etudes sur «Le Fils naturel » et les «Entretiens sur le Fils naturel » de Diderot, Oxford, ViF, Diderot Studies, 29, p. 205.

Conroy (W.), 1975, Diderot's «Essai sur Sénèque », Studies in Voltaire and the Eighteenth Century, 131, Oxford, The Voltaire Foundation.

DENT (Nicholas J. H.), 1996, «Vertu, éthique de la vertu » dans Dictionnaire d'éthique et de philosophie morale, éd. Monique Canto-Sperber, Paris, Presses universitaires de France, p. 1665-72. 
DERRIDA (Jacques), 2003, Chaque fois unique la fin du monde, Paris, Galilée.

Derrida (J.), 2004, Politiques de l'amitié, Paris, Galilée.

DiDEROT (Denis), 1778, Essai sur la vie de Sénèque le philosophe, sur ses écrits et sur les règnes de Claude et de Néron, Paris, De Bure.

DIDEROT (D.), 1782, Essai sur les règnes de Claude et de Néron et sur les mours et les écrits de Sénèque pour servir à l'introduction de la lecture de ce philosophe, 2 tomes, Londres, sans nom d'éditeur.

Diderot (D.), 1875-77, Euvres complètes, éd. Jules Assézat et Maurice Tourneux, 20 tomes, Paris, Garnier.

Diderot (D.), 1994-99, Euvres, éd. Laurent Versini, 5 tomes, Paris, Robert Laffont.

EHRARD (Jean), 1995, « Pourquoi Sénèque? », dans DIDEROT, (D.), 1975-, Euvres complètes, éd. Herbert Dieckmann, Jacques Proust et Jean Varloot, Paris, Hermann, vol. XXV, p. 3-18.

FERRET (Olivier), 2007, La fureur de nuire : échanges pamphlétaires entre philosophes et antiphilosophes, 1750-1770, Oxford, The Voltaire Foundation, 2007: 03.

FouCAUlT (Michel), 1962, «Introduction », dans Jean-Jacques Rousseau, Rousseau juge de Jean-Jacques. Dialogues, Paris, A. Colin, p. vii-xxiv.

Fowler (James), 2013, «État présent : Diderot », French Studies, 67.3, Oxford, Oxford University Press, p. 386-393.

Fumaroli (Marc), 2015, Quand l'Europe cultivait les lettres, Paris, Gallimard.

GARAT (Doninique Joseph), 1779, «Lettre aux Auteurs du Journal de paris, sur la Notice qu'ils ont donné de la Vie de Sénèque », Mercure de France, 15 février, p. 172-190.

GATEFIN (Eric), 2007, Diderot, Sénèque et Jean-Jacques. Un dialogue à trois voix, Amsterdam, Rodopi.

GOODMAN (Jessica), et HARRIS, (Joseph), 2018, Anticipated Afterlives : Envisaging Posterity in Early Modern France, numéro spécial de la revue Early Modern French Studies.

Goulemot (Jean-Marie), 2012, « La vieillesse des philosophes : le cas Diderot », Diderot Studies, 32, Genève, Droz, p. 165-80.

GRIMM (Friedrich Melchior), 1880, «Essai sur Sénèque », dans GRIMM (F. M.), (éd.), 188782, Correspondance littéraire, philosophique et critique, éd. Maurice Tourneux, 16 tomes, Paris, Garnier.

HAYES (Julie Candler), 2009, «Aspects du style tardif dans l'Essai sur les règnes de Claude et de Néron », Diderot Studies, 31, Genève, Droz, p. 37-44.

HINDE STEWART (Joan), 2010, The Enlightenment of Age: Women, Letters and Growing Old in Eighteenth-century France, SVEC, Oxford, The Voltaire Foundation, 2010 $: 09$.

LECA-TSIOMIS, (Marie), 1999, Écrire l'Encyclopédie. Diderot : de l'usage des dictionnaires à la grammaire philosophique, Studies in Voltaire and the Eighteenth Century, Oxford, The Voltaire Foundation, 375.

LECA-TSIOMIS (M.), 2004, «Diderot et le nom d'ami : à propos de l'Essai sur les règnes de Claude de Néron », Recherches sur Diderot et l'Encyclopédie, 36, Langres, Société Diderot, p. 97-108.

LiLti (Antoine), 2014, Figures publiques: Invention de la célébrité (1750-1800), Paris, Fayard.

MALl (Laurence), 2000 «Une auto-biolecture : l'Essai sur les règnes de Claude et de Néron de Diderot », Diderot Studies, 28, Genève, Droz, p. 111-122.

MALl (L.), 2004, « Sénèque et Diderot, sujets à caution dans l'Essai sur les règnes de Claude et de Néron », Recherches sur Diderot et l'Encyclopédie, 36, Langres, Société Diderot, p. 4356.

MARMONTEl (Jean), 1778, «Extrait d'un essai sur la Vie de Sénèque le philosophe », 
Mercure de France, 15 décembre, p. 136-160.

Marmontel (J.), 1778a, «Suite de l'Essai sur la Vie de Sénèque le philosophe », Mercure de France, 25 décembre, p. 275-294.

NouIS (Lucien), 2010, « L'emploi du temps : Diderot et Rousseau, lecteurs de Sénèque », French Studies, 64.2, Oxford, Oxford University Press, p. 150-163.

MARKovits (Rahul), 2009, "L'incendie de la comédie de Genève (1768): Rousseau, Voltaire et l'impérialisme culturel français », Revue historique, 4, Paris, Presses Universiatires de France, p. 831-873.

MASSEAU (Didier), 2000, Les ennemis des philosophes : l'anti-philosophie au temps des Lumières, Paris, Albin Michel.

MAsseau (D.), 2012, «L'enjeu d'une polémique : la figure de Sénèque dans i'Essai sur les règnes de Claude et de Néron », Diderot Studies, XXXII, Genève, Droz, p. 149-164.

NusSBAUM (Martha), 1998, Cultivating Humanity : a classical defense of reform in liberal education, Cambridge Mass., Harvard University Press.

Roudet (Nicolas), 2015, «Quand l'Europe cultivait l'esprit », Acta fabula, vol. 16, n 6 , Essais critiques, septembre-octobre 2015, URL : http://www.fabula.org/revue/document9498.php (dernière consultation 5/7/2016).

ROUSSEAU (Jean-Jacques), 1995, Les Confessions, Paris, Gallimard.

Rousseau (J.-J.), 1965-95, Correspondance complète de Jean-Jacques Rousseau, éd. R. A. Leigh, 51 tomes, Genève, Institut et Musée Voltaire.

SENEQUe, 1778, Les Euvres de Sénèque le philosophe, traduites en français par feu M. Lagrange avec des notes de critique, d'histoire et de literature, Paris, De Bure.

STALnAKER (Joanna), 2009, " Diderot's Literary Testament », Diderot Studies, 31, Genève, Droz, p. 45-56.

STENGER (Gerhardt), 2004, L'affaire des Cacouacs : trois pamphlets contre les philosophes des lumières, Saint-Etienne, Publications de l'université de Saint-Etienne.

Trousson (Raymond), 2006, Diderot jour après jour, Paris, Champion.

Trousson (R.), 2000, Jean-Jacques Rousseau jugé par ses contemporains, Paris, Champion. Tunstall, (Kate E.), 2014, compte rendu de VINCENT, (Charles), 2014, H-France. http://www.h-france.net/vol15reviews/vol15no54tunstall.pdf (dernière consultation 5/7/2016). Tunstall (K. E.) et ANDERSON (Wilda), éds., 2013, Naming, Renaming, and Unnaming in Early Modern and Enlightenment Europe, numéro spécial double de Romance Studies, 31-34.

Tunstall (K. E.), 2002, «Dossier», dans CRONK, (Nicholas), (éd.) Études sur «Le Fils naturel » et les «Entretiens sur le Fils naturel » de Diderot, Oxford, ViF, p. 211-324.

VINCENT (Charles), 2014, Diderot en quête d'éthique, Paris, Garnier.

WaCJMAN (Claude), 1996, Les Jugements de la critique sur la "folie » de Jean-Jacques Rousseau: représentations et interprétations, 1760-1990, Studies in Voltaire and the Eighteenth Century, 337, Oxford, The Voltaire Foundation. 